\title{
IN SILICO DOCKING ANALYSIS OF YOHIMBINE ALKALOIDS FROM CATHARANTHUS ROSEUS AGAINST DENGUE FEVER
}

\author{
GIRIJA R $\mathbf{R}^{1}$, ARUNA $S^{2}$, SANGEETHA $\mathbf{R}^{1 *}$ \\ ${ }^{1}$ Bioinformatics Infrastructure Facility Centre, Queen Mary's College, Chennai, Tamil Nadu, India. ${ }^{1,2}$ Department of Chemistry, \\ Queen Mary's College, Chennai, Tamil Nadu, India. Email: sangeetharajendran088@gmail.com
}

Received: 26 June 2019, Revised and Accepted: 30 March 2019

\section{ABSTRACT}

Objective: In this docking study was performed by the active site of NS2B-NS3 protease with selected plant alkaloids derived from Catharanthus roseus. On the basis of docking results, out of the nine alkaloids yohimbine might be considered as the best inhibitors of NS2B-NS3 protease protein.

Methods: Lipinski rule was employed to check the ligand likeliness of the compound. The three-dimensional crystallographic structure of NS2B-NS3 protease (ID.2FOM) fetched from the protein data bank. The in silico docking studies were performed by "AutoDock version 4.2" software tool.

Results: Yohimbine is having the best binding score $(-7.18 \mathrm{Kcal} / \mathrm{mol})$ than the other eight compounds.

Conclusion: Hence, it has been concluded yohimbine as a novel inhibitor for NS2B-NS3 protease in dengue fever.

Keywords: NS2B-NS3 protease, Catharanthus roseus, Yohimbine, Protein data bank, AutoDock.

(C) 2019 The Authors. Published by Innovare Academic Sciences Pvt Ltd. This is an open access article under the CC BY license (http://creativecommons. org/licenses/by/4. 0/) DOI: http://dx.doi.org/10.22159/ajpcr.2019.v12i5.32815

\section{INTRODUCTION}

Catharanthus roseus which is an important medicinal plant of the family Apocynaceae used to treat many fatal diseases.

Kingdom: Plantae

Phylum: Magnoliophyta

Class: Magnoliopsida

Order: Gentianales

Family: Apocynaceae

Genus: Catharanthus

Species: Roseus

Binomial name: Catharanthus roseus $\mathrm{L}$.

C. roseus also possesses good antioxidant potential. There are about two common cultivars of $C$. roseus which is named on the basis of their flower color, flower that is the pink flowered is termed as "rosea" and white color flowered is termed as "alba." In India, it is known as "Nithyakalyani" [1]. C. roseus is an ornamental shrub that grows up to $30-100 \mathrm{~cm}$ in height. This plant has a long history as a folk medicine in many countries such as South Africa, China, India, Mexico, and Malaysia where it is utilized as a remedy to alleviate diabetes complications [2]. C. roseus plant parts such as root, stem, leaves, and flowers produce several phytoactive compounds such as flavonoids, alkaloids, steroids, terpenoids, tannin, phenolics, anthocyanins, fatty acids, proteins, phytohormones, and enzymes [3]. The other medicinal properties of C. roseus include properties such as antibacterial, antifungal, antiviral, antidiabetic, and anticancer activities [4]. The various alkaloids found to report have vindoline, vindolinine, vindolidin, tabersonine, ajmalicine, lochnericine, horhammericine, yohimbine, and secologanin [5]. Vinblastine and vincristine are the most important antitumor bisindole alkaloids, which are derived from the coupling of vindoline and catharanthine monomers. The biochemical synthesis of six steps enzymatic conversion of tabersonine to vindoline has been studied extensively. The terminal step of vindoline biosynthesis is catalyzed by deacetyl vindoline-4-0 acetyltransferase [6].
The cause of dengue fever is Aedes mosquitoes (Aedes aegypti). It is a viral disease and transmitted between human hosts. Every year more than 20 million cases of dengue fever recorded in the whole world. The dengue viruses (DVs) are members of the genus Flavivirus in the family Flaviviridae [7]. The signs and symptoms of dengue fever include high fever, up to $105^{\circ} \mathrm{F}$, severe headache, retro-orbital (behind the eye) pain, severe joint and muscle pain, nausea and vomiting, and rash. The rash may appear over most of the body 3-4 days after the fever begins, and then subsides after 1-2 days. There may be a second rash a few days later. These symptoms include high fever, up to $105^{\circ} \mathrm{F}$, severe headache, retro-orbital (behind the eye) pain, severe joint and muscle pain, nausea and vomiting, and rash.

Symptoms of dengue hemorrhagic fever include all of the symptoms of classic dengue plus bleeding from the nose, gums, or under the skin, causing purplish bruises, which results from damage to blood vessels. This type of dengue disease can cause death. Dengue vaccines have been under development since the 1940s, but a tetravalent vaccine which simultaneously provides long-term protection against all DV serotypes is around the corner. A tetravalent antigen was designed by splicing the EDIIIs of DV-1, DV-2, DV-3, and DV-4 using flexible pentaglycyl linkers [8]. At present, there are no treatment options for dengue fever; there is no known cure or vaccine. There are several DV proteins that have been identified as potential drug targets [9]. Natural products have been and continue to be excellent sources of medicinal agents, as themselves or as templates for synthetic agents [10]. The people of developing nations often have limited access to health care and inadequate resources for pharmaceuticals, and traditional herbal medicines offer the potential for treatments of diseases in these regions. In this work, we have screened our virtual library of phytochemicals against several protein targets of DV, included DV protease (NS2BNS3protease) using a molecular docking approach [11].

\section{DV protease}

DENV protease (NS2B-NS3pro) is a trypsin-like serine protease that cleaves the dengue polyprotein into individual proteins necessary for viral replication. DENV protease has been identified as a primary target 
for the development of dengue antiviral drugs [12]. There are two potential targets for DENV protease inhibition: (1) The active site of the protease [13] and (2) block association of NS3 protease with its protein cofactor NS2B [14].

\section{DATABASE AND METHODOLOGY}

Preparation of receptor structure

The protein data bank (PDB) is a crystallographic database for threedimensional (3D) structure data of large biological molecules such as proteins and nucleic acids. Crystal structure of NS2B-NS3 protease was obtained from PDB (http://www.rcsb.org) with PDB ID 2FOM. The protein consists of two chains and 185 residues length with resolution $1.5 \AA$ A. Structural and active site studies of the protein were done using PyMol molecular visualization software $[15,16]$.

\section{PubChem}

It is a product of the NCBI database. It is most useful for collecting the information about the specified chemicals. It is an online archive containing the information of all the known chemicals their properties and their biological importance. The user can use these chemicals based on their function and download their structures for other analysis. The two-dimensional (2D) structure of the C. roseus compounds of vindoline, vindolinine, vindolidin, tabersonine, ajmalicine, lochnericine, horhammericine, yohimbine, and secologanin was obtained from the PubChem database [17].

\section{ACD ChemSketch}

$\mathrm{ACD} /$ ChemSketch is the powerful chemical drawing and graphics package from ACD/Labs software. Draw the chemical structures and calculate chemical properties are very quickly and easily [18]. The 3D structures of vindoline, vindolinine, vindolidin, tabersonine, ajmalicine, lochnericine, Horhammericine, yohimbine, and secologanin were drawn by ChemSketch.

\section{Open Babel}

Open Babel is software that is used to inter convert chemical file format. It is open access and collaborative project allows to search, convert, analyze all store data from molecular modeling, chemistry, solid-state materials, biochemistry, or related areas (www.openbabel.org).

\section{Ligand structure preparation}

In this study, for the ligand vindoline, vindolinine, vindolidin, tabersonine, ajmalicine, lochnericine, horhammericine, yohimbine, and secologanin the 2D structure and chemical formula were obtained from PubChem. The PubChem ID of Vindoline (260535), Vindolinine (24148538), vindolidin (11618751), tabersonine (20485), ajmalicine (441975), Lochnericine (11382599), horhammericine (443358), yohimbine (8469), and secologanin (91302444) were obtained. The structure of vindoline, vindolinine, vindolidin, tabersonine, ajmalicine, lochnericine, horhammericine, yohimbine, and secologanin was computed by drawing using ChemSketch software (chemically intelligent drawing interface freeware software). This was followed by ligand construction using ChemSketch draw mode 3D structure optimization were compute and finally ligand as "MOL" file format and then mol file was converted to "PDB" using open babel software. The protein and ligand files which are prepared were then taken for docking [17].

\section{Docking methodology}

The ligand was docked to the active site of the target (NS2B-NS3 protease) using AutoDock program, version 4.2 [19]. AutoDock is a suite of automated docking tools. The software is used for modeling flexible small molecules such as drug molecules and its binding to receptor proteins of known 3D structures. It uses genetic algorithms for the conformational search and is a suitable method for docking studies. This technique is based upon, grid based method of energy evaluation. AutoDock tools are used to prepare, run and analyze the docking simulations and modeling studies. After the grid generation, the processed ligand was docked with the protein to evaluate the interaction between each target protein and ligand. Interaction was hydrophilic, Van der Waals, and hydrophobic. The interaction strength varies from protein to protein based out its affinity procedure, the ligand. During the autodocking procedure, the ligand confirmation was retained followed by the extra precision mode selection $[18,20]$.

\section{Visualization and analyzing docking results}

PyMol is an open source tool to visualize molecules available from (www.pymol.org). PyMol has excellent capabilities in creating highquality images from 3D structures; it has well-developed functions for manipulating structures and some basic functions to analyze their chemical properties. The possibilities to write scripts and plugin as well as to incorporate PyMol in custom software are fast and superior to most other programs. Once the target ligand was docked against all protein of interest, the result was visualized for their interactions, binding energy, H-bond formation, and few other parameters using the PyMol software $[17,18]$

\section{RESULTS AND DISCUSSION}

\section{Structure retrieval}

The 3D crystallographic structure of NS2B-NS3Protease (ID.2FOM) were downloaded from the PDB.

\section{Preparation of ligand}

For further docking analysis of the compounds vindoline, vindolinine, vindolidin, tabersonine, ajmalicine, lochnericine, horhammericine, yohimbine, and secologanin from C. roseus are taken. The 2D structures of the ligand were generated using the ACD/ChemSketch tool. This tool contains for 2D cleaning, 3D optimization, and viewing. These data are saved as a molecular format file (molecular design limited and MOL format). The molecular format converter tool (open babel) is used to convert this file into the PDB format and is used during docking analysis. The structure and molecular formula of vindoline, vindolinine, vindolidin, tabersonine, ajmalicine, lochnericine, horhammericine, yohimbine, and secologanin compounds [18] was shown in Table 1.

According to Lipinski's rule of a compound having not more than 5 hydrogen bond donors $(\mathrm{OH}$ an $\mathrm{NH}$ groups), not more than 10 hydrogen bond acceptors (notably $\mathrm{N}$ and $\mathrm{O}$ ), molecular weight under $500 \mathrm{~g} / \mathrm{mol}$, partition coefficient $\log \mathrm{P}$ of $<5[18]$ is shown in Table 2.

\section{Docking study}

On the basis of molecular docking studies, was usually smaller molecule which binds with the larger molecule. The inhibitors docked with targeted DV protein of NS2BNS3 protease (ID.2FOM) using AutoDock software (Version 4.2). The graphical user interface program "AutoDock Tools" was used to prepare, run, and analyze the docking simulations. Kollman united atom charges, solvation parameters, and polar hydrogens were added into the receptor PDB file for the preparation of protein in docking simulation [17]. AutoDock results were analyzed to study the interactions and the binding energy of the docked structure [21]. The best ligand-receptor structure from the docked structures was chosen based on the lowest energy and minimal solvent accessibility of the ligand. The docking results were visualized using the PyMol visualizer tool [22]. These structure and results are shown in the form of the figures and tables.

The alkaloid compound yohimbine is docked against NS2B-NS3 protease. Kollman united atom charges, salvation parameters and polar hydrogens were added into the receptor PDB file for the preparation of protein in docking simulation requires precalculated grid maps, each atom type present in the flexible molecules being docked and its stores the potential energy. This grid box must surround the macromolecule. The grid box size was set at 126,126 , and $126 \AA$ ( $x, y$, and $\mathrm{z}$ ) to include all the amino acid residues that present rigid macromolecules. Auto grid 4.2 program, supplied with AutoDock was used to produce grid maps. The spacing between grid points was $0.375 \AA$ A. The Lamarckian genetic algorithm [20] was chosen to search for the best conformers. 
Table 1: 2D and 3D structure of $C$. roseus

Compound name

Vindoline

Vindolinine

Ajmalicine

Tabersonine

Lochnericine

Horhammericine

Yohimbine

$\mathrm{C}_{21} \mathrm{H}_{26} \mathrm{~N}_{2} \mathrm{O}_{3}$

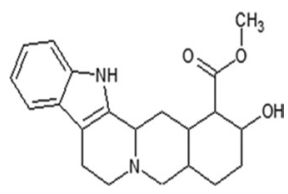

Vindolidin

Molecular formula

$\mathrm{C}_{25} \mathrm{H}_{32} \mathrm{~N}_{2} \mathrm{O}_{6}$

$\mathrm{C}_{21} \mathrm{H}_{24} \mathrm{~N}_{2} \mathrm{O}_{2}$

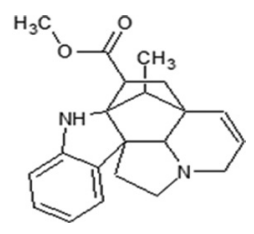

$\mathrm{C}_{21} \mathrm{H}_{24} \mathrm{~N}_{2} \mathrm{O}_{3}$

$\mathrm{C}_{21} \mathrm{H}_{24} \mathrm{~N}_{2} \mathrm{O}_{2}$

$\mathrm{C}_{21} \mathrm{H}_{24} \mathrm{~N}_{2} \mathrm{O}_{3}$

$\mathrm{C}_{21} \mathrm{H}_{24} \mathrm{~N}_{2} \mathrm{O}_{4}$
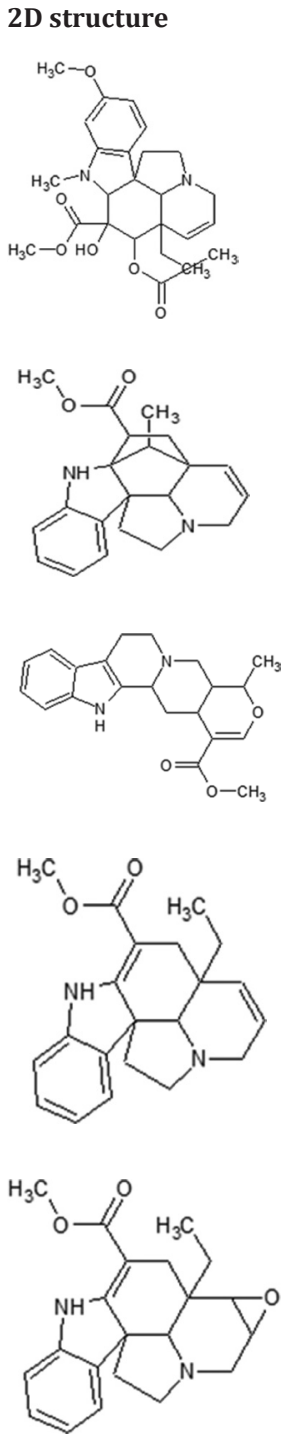
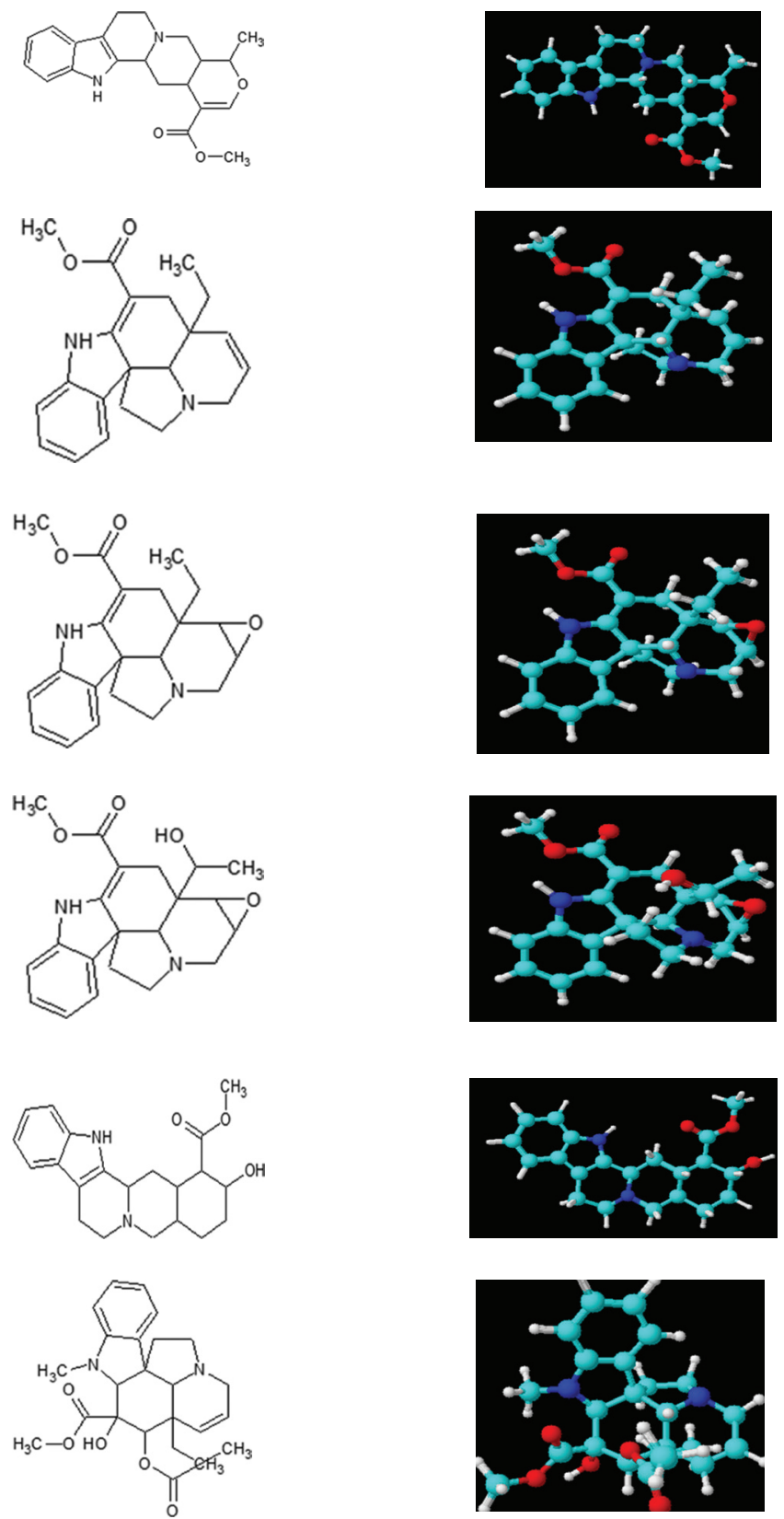

3D structure
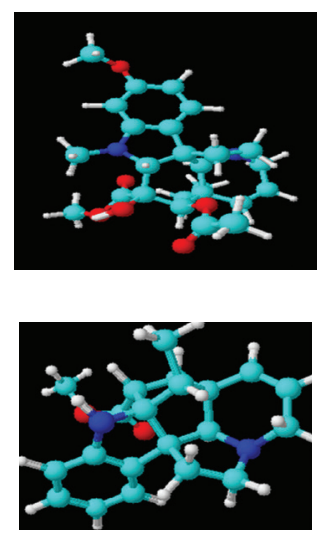

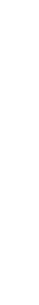


Table 1: (Continued)

\begin{tabular}{lll}
\hline Compound name & Molecular formula & 3D structure \\
\hline Secologanin & $\mathrm{C}_{17} \mathrm{H}_{24} \mathrm{O}_{10}$ & \\
\hline
\end{tabular}

C. roseus: Catharanthus roseus, 2D: Two-dimensional, 3D: Three-dimensional

Table 2: Lipinski's rule of compounds

\begin{tabular}{llllll}
\hline Compound name & Molecular formula & Molecular weight $(\mathbf{g} / \mathbf{m o l})$ & Donor & Acceptor & Log value \\
\hline Vindoline & $\mathrm{C}_{25} \mathrm{H}_{32} \mathrm{~N}_{2} \mathrm{O}_{6}$ & 456.539 & 1 & 8 & 1.3 \\
Vindolinine & $\mathrm{C}_{21} \mathrm{H}_{24} \mathrm{~N}_{2} \mathrm{O}_{2}$ & 336.435 & 1 & 4 & 2.9 \\
Ajmalicine & $\mathrm{C}_{21} \mathrm{H}_{24} \mathrm{~N}_{2} \mathrm{O}_{3}$ & 352.434 & 1 & 4 & 2.7 \\
Tabersonine & $\mathrm{C}_{21} \mathrm{H}_{24} \mathrm{~N}_{2} \mathrm{O}_{2}$ & 336.435 & 1 & 4 & 3.4 \\
Lochnericine & $\mathrm{C}_{21} \mathrm{H}_{24} \mathrm{~N}_{2} \mathrm{O}_{3}$ & 352.434 & 1 & 5 & 2.5 \\
Horhammericine & $\mathrm{C}_{21} \mathrm{H}_{24} \mathrm{~N}_{2} \mathrm{O}_{4}$ & 368.433 & 2 & 6 & 1.4 \\
Yohimbine & $\mathrm{C}_{21} \mathrm{H}_{26} \mathrm{~N}_{2} \mathrm{O}_{3}$ & 354.455 & 1 & 4 & 7 \\
Vindolidin & $\mathrm{C}_{24} \mathrm{H}_{30} \mathrm{~N}_{2} \mathrm{O}_{5}$ & 426.513 & 4 & 10 & 1.9 \\
Secologanin & $\mathrm{C}_{17} \mathrm{H}_{24} \mathrm{O}_{10}$ & 388.369 & & & 1.6 \\
\hline
\end{tabular}

Table 3: Docking interaction between NS2B-NS3 protease and vindoline

\begin{tabular}{lllll}
\hline NS2B-NS3 protease & & & Vindoline & Distance A \\
\cline { 2 - 2 } Residue & Atom & Atom & Binding score (Kcal/mol) \\
\hline GLY 153 & $\mathrm{N}$ & 0 & 3.1 & -6.14 \\
GLY 153 & 0 & 0 & 2.7 & \\
\hline
\end{tabular}

Table 4: Docking interaction between NS2B-NS3 protease and vindolidin

\begin{tabular}{llllll}
\hline NS2B-NS3 protease & & & Vindolidin & Distance $\AA$ & Binding score (Kcal/mol) \\
\cline { 1 - 2 } Residue & Atom & & Atom & & -5.64 \\
\hline GLY 153 & $\mathrm{N}$ & 0 & 3.1 & \\
GLY 153 & $\mathrm{N}$ & 0 & 3.2 & \\
GLY 151 & O & 0 & 3.4 \\
ASP 75 & OD2 & ND1 & 0 & 2.7 & \\
HIS 51 & & 0 & 3.0 & \\
\hline
\end{tabular}

Table 5: Docking interaction between NS2B-NS3 protease and vindolinine

\begin{tabular}{|c|c|c|c|c|}
\hline \multicolumn{2}{|c|}{ NS2B-NS3 protease } & \multirow{2}{*}{$\begin{array}{l}\text { Vindolinine } \\
\text { Atom }\end{array}$} & \multirow[t]{2}{*}{ Distance Å } & \multirow[t]{2}{*}{ Binding score $(\mathrm{Kcal} / \mathrm{mol})$} \\
\hline Residue & Atom & & & \\
\hline LYS 74 & NZ & 0 & 2.7 & -8.09 \\
\hline
\end{tabular}

Table 6: Docking interaction between NS2B-NS3 protease and ajmalicine

\begin{tabular}{|c|c|c|c|c|}
\hline \multicolumn{2}{|c|}{ NS2B-NS3 protease } & \multirow{2}{*}{$\begin{array}{l}\text { Ajmalicine } \\
\text { Atom }\end{array}$} & \multirow[t]{2}{*}{ Distance Å } & \multirow[t]{2}{*}{ Binding score (Kcal/mol) } \\
\hline Residue & Atom & & & \\
\hline PRO 132 & 0 & $\mathrm{~N}$ & 2.9 & -6.21 \\
\hline TYR 150 & $\mathrm{OH}$ & 0 & 3.4 & \\
\hline SER 135 & OG & 0 & 2.8 & \\
\hline
\end{tabular}

The alkaloid compound yohimbine and NS2B-NS3 protease binding energy are shown in Fig. 9a and $\mathrm{b}$ and the interactions visualization using the PyMol visualizer. The docking study was performed between yohimbine compounds from $C$. roseus against NS2B-NS3 protease in 
Table 7: Docking interaction between horhammericine against NS2B-NS3 protease

\begin{tabular}{|c|c|c|c|c|}
\hline \multicolumn{2}{|c|}{ NS2B-NS3protease } & \multirow{2}{*}{$\begin{array}{l}\text { Horhammericine } \\
\text { Atom }\end{array}$} & \multirow[t]{2}{*}{ Distance Å } & \multirow[t]{2}{*}{ Binding score (Kcal/mol) } \\
\hline Residue & Atom & & & \\
\hline GLY 153 & $\mathrm{~N}$ & 0 & 2.8 & -6.55 \\
\hline SER 135 & NE2 & 0 & 2.8 & \\
\hline SER 135 & OG & 0 & 3.2 & \\
\hline
\end{tabular}

Table 8: Docking interaction between NS2B-NS3 protease and lochnericine

\begin{tabular}{|c|c|c|c|c|}
\hline \multicolumn{2}{|c|}{ NS2B-NS3 protease } & \multirow{2}{*}{$\begin{array}{l}\text { Lochnericine } \\
\text { Atom }\end{array}$} & \multirow[t]{2}{*}{ Distance $\AA$} & \multirow[t]{2}{*}{ Binding score (Kcal/mol) } \\
\hline Residue & Atom & & & \\
\hline SER 135 & $\mathrm{OG}$ & $\mathrm{N}$ & 2.7 & -6.19 \\
\hline SER 135 & OG & 0 & 2.8 & \\
\hline PRO 132 & 0 & $\mathrm{~N}$ & 3.0 & \\
\hline PRO 132 & 0 & 0 & 3.2 & \\
\hline TYR 150 & $\mathrm{OH}$ & 0 & 2.8 & \\
\hline
\end{tabular}

Table 9: Docking interaction between NS2B-NS3 protease and secologanin

\begin{tabular}{|c|c|c|c|c|}
\hline \multicolumn{2}{|c|}{ NS2B-NS3 protease } & \multirow{2}{*}{$\begin{array}{l}\text { Secologanin } \\
\text { Atom }\end{array}$} & \multirow[t]{2}{*}{ Distance $\AA$} & \multirow[t]{2}{*}{ Binding score (Kcal/mol) } \\
\hline Residue & Atom & & & \\
\hline LYS 73 & 0 & 0 & 3.5 & -6.95 \\
\hline LYS 73 & 0 & 0 & 2.9 & \\
\hline ASN 152 & ND2 & 0 & 3.3 & \\
\hline ASN 152 & ND2 & 0 & 2.6 & \\
\hline ASN 152 & ND2 & 0 & 3.4 & \\
\hline LEU 149 & $\mathrm{~N}$ & 0 & 2.7 & \\
\hline LEU 149 & $\mathrm{~N}$ & 0 & 3.1 & \\
\hline TRP 83 & NE1 & 0 & 3.1 & \\
\hline
\end{tabular}

Table 10: Docking interaction between NS2B-NS3 protease and tabersonine

\begin{tabular}{|c|c|c|c|c|}
\hline \multicolumn{2}{|c|}{ NS2B-NS3 protease } & \multirow{2}{*}{$\begin{array}{l}\text { Tabersonine } \\
\text { Atom }\end{array}$} & \multirow[t]{2}{*}{ Distance $\AA ̊$} & \multirow[t]{2}{*}{ Binding score (Kcal/mol) } \\
\hline Residue & Atom & & & \\
\hline GLY151 & 0 & $\mathrm{~N}$ & 3.0 & -6.57 \\
\hline GLY151 & 0 & 0 & 2.6 & \\
\hline GLY153 & $\mathrm{N}$ & 0 & 2.8 & \\
\hline ASP 75 & OD2 & 0 & 3.2 & \\
\hline
\end{tabular}

Table 11: Docking interaction between NS2B-NS3 protease and yohimbine

\begin{tabular}{|c|c|c|c|c|}
\hline \multicolumn{2}{|c|}{ NS2B-NS3 protease } & \multirow{2}{*}{$\begin{array}{l}\text { Yohimbine } \\
\text { Atom }\end{array}$} & \multirow[t]{2}{*}{ Distance $\AA ̊$} & \multirow[t]{2}{*}{ Binding score (Kcal/mol) } \\
\hline Residue & Atom & & & \\
\hline PHE 130 & 0 & 0 & 2.7 & -7.18 \\
\hline TYR 150 & $\mathrm{OH}$ & 0 & 3.0 & \\
\hline TYR 150 & $\mathrm{OH}$ & 0 & 3.4 & \\
\hline SER 135 & OG & 0 & 2.6 & \\
\hline GLY 151 & 0 & $\mathrm{~N}$ & 3.1 & \\
\hline
\end{tabular}

Table 12: Overall docking result of NS2B-NS3 protease

\begin{tabular}{|c|c|c|c|c|}
\hline S. No. & Compound name & Key residue & Docking energy (Kcal/mol) & No. of. hydrogen bonds \\
\hline 1 & Vindoline & GLY 153, GLY 153 & -6.14 & 2 \\
\hline 2 & Vindolinine & LYS 74 & -8.09 & 1 \\
\hline 3 & Ajmalicine & PRO 132, TYR 150, SER 135 & -6.21 & 0 \\
\hline 4 & Tabersonine & GLY151, GLY151, GLY153, ASP 75 & -6.57 & 1 \\
\hline 5 & Lochnericine & SER 135, SER 135, PRO 132, PRO 132, TYR 150 & -6.19 & 1 \\
\hline 6 & Horhammericine & GLY 153, HIS 51, SER 135, SER 135 & -6.55 & 1 \\
\hline 7 & Yohimbine & PHE 130 , TYR 150, TYR 150, SER 135, GLY 151 & -7.18 & 3 \\
\hline 8 & Vindolidin & GLY 153, GLY 153, GLY 151, ASP 75, HIS 51 & -5.64 & 1 \\
\hline 9 & Secologanin & $\begin{array}{l}\text { LYS 73, LYS 73, ASN 152, ASN 152, ASN 152, } \\
\text { LEU 149, LEU 149, TRP } 83\end{array}$ & -6.95 & 2 \\
\hline
\end{tabular}




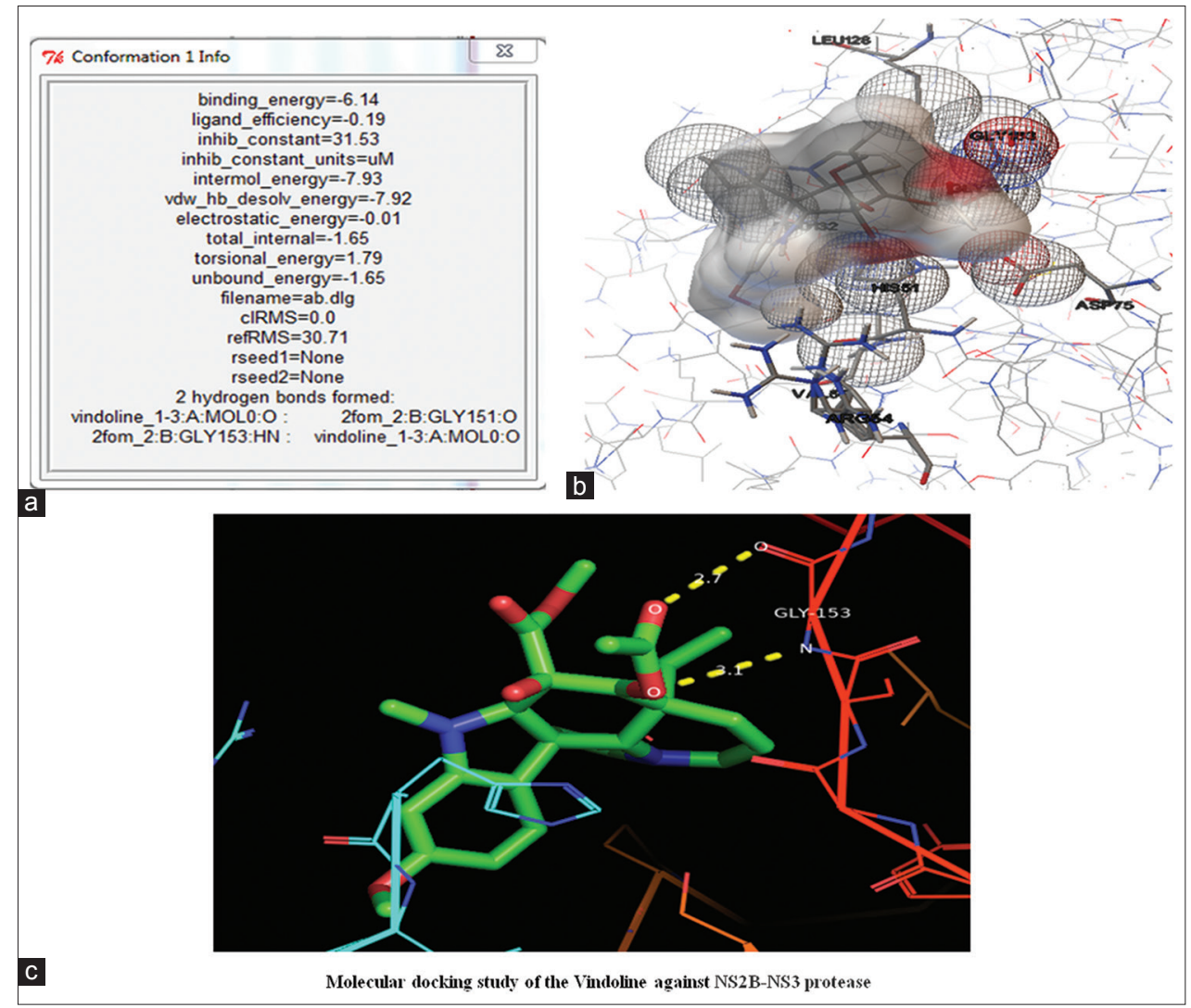

Fig. 1: Docking of NS2B-NS3 protease and vindoline (a) binding energy (b) interaction between NS2B-NS3 protease and vindoline as visualized using AutoDock (c) hydrogen bond forms between NS2B-NS3 protease and vindoline is visualized using PyMol

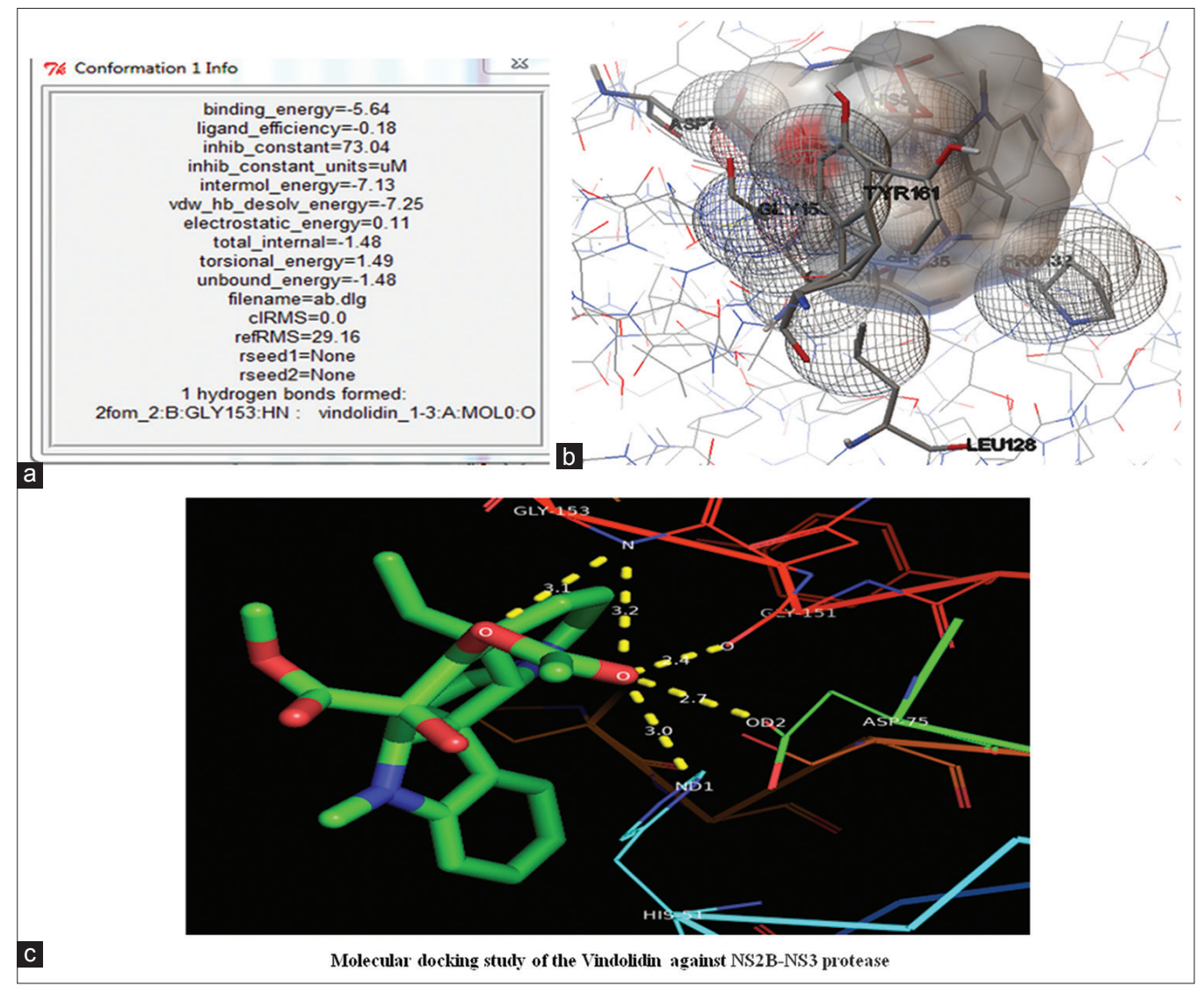

Fig. 2: Docking of NS2B-NS3 protease and vindolidin (a) binding energy (b) interaction between NS2B-NS3 protease and vindolidin as visualized using AutoDock (c) hydrogen bond forms between NS2B-NS3 protease and vindolidin is visualized using PyMol 


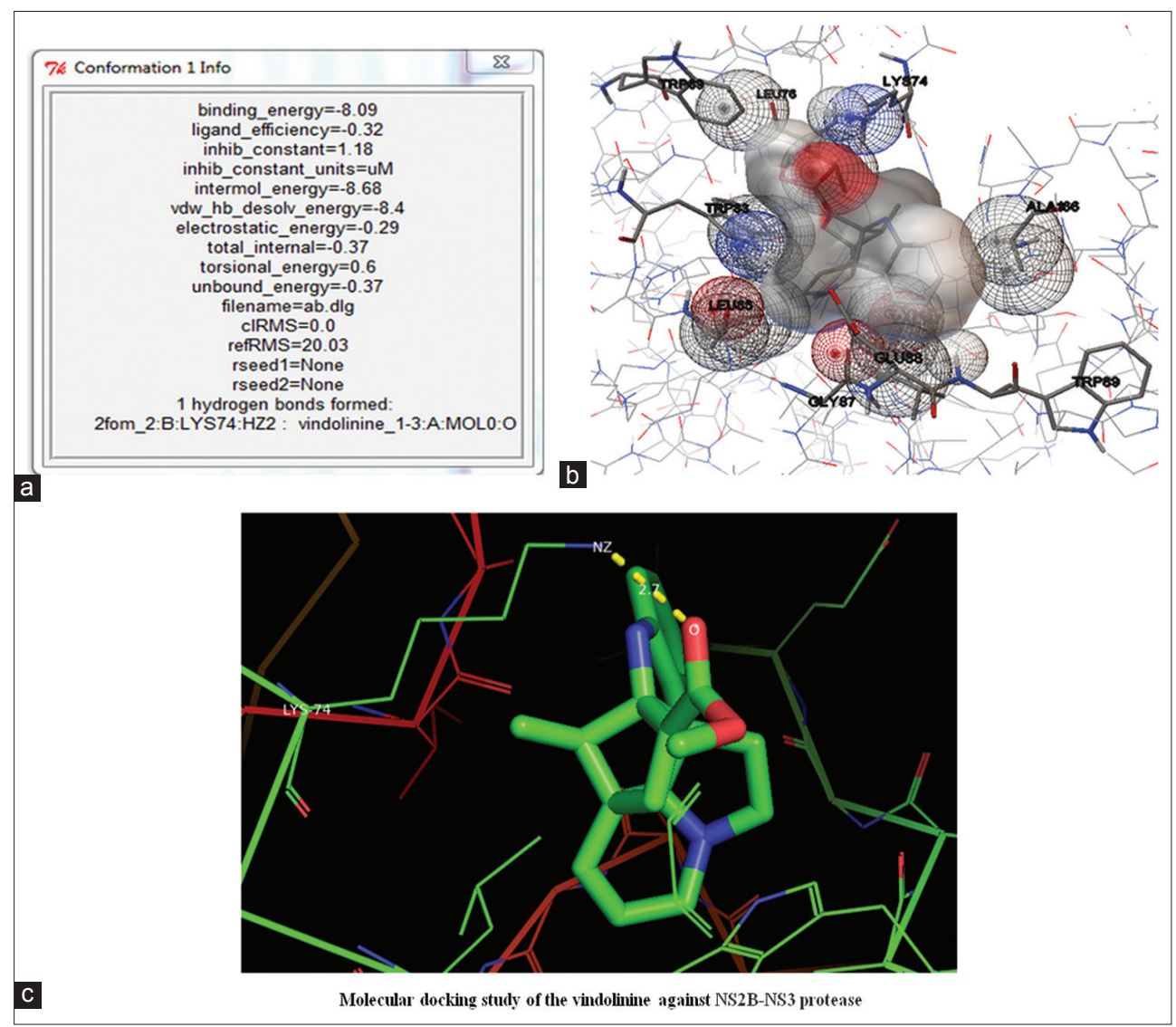

Fig. 3: Docking of NS2B-NS3 protease and vindolinine (a) binding energy (b) interaction between NS2B-NS3 protease and vindolinine as visualized using AutoDock (c) hydrogen bond forms between NS2B-NS3 protease and vindolinine is visualized using PyMol

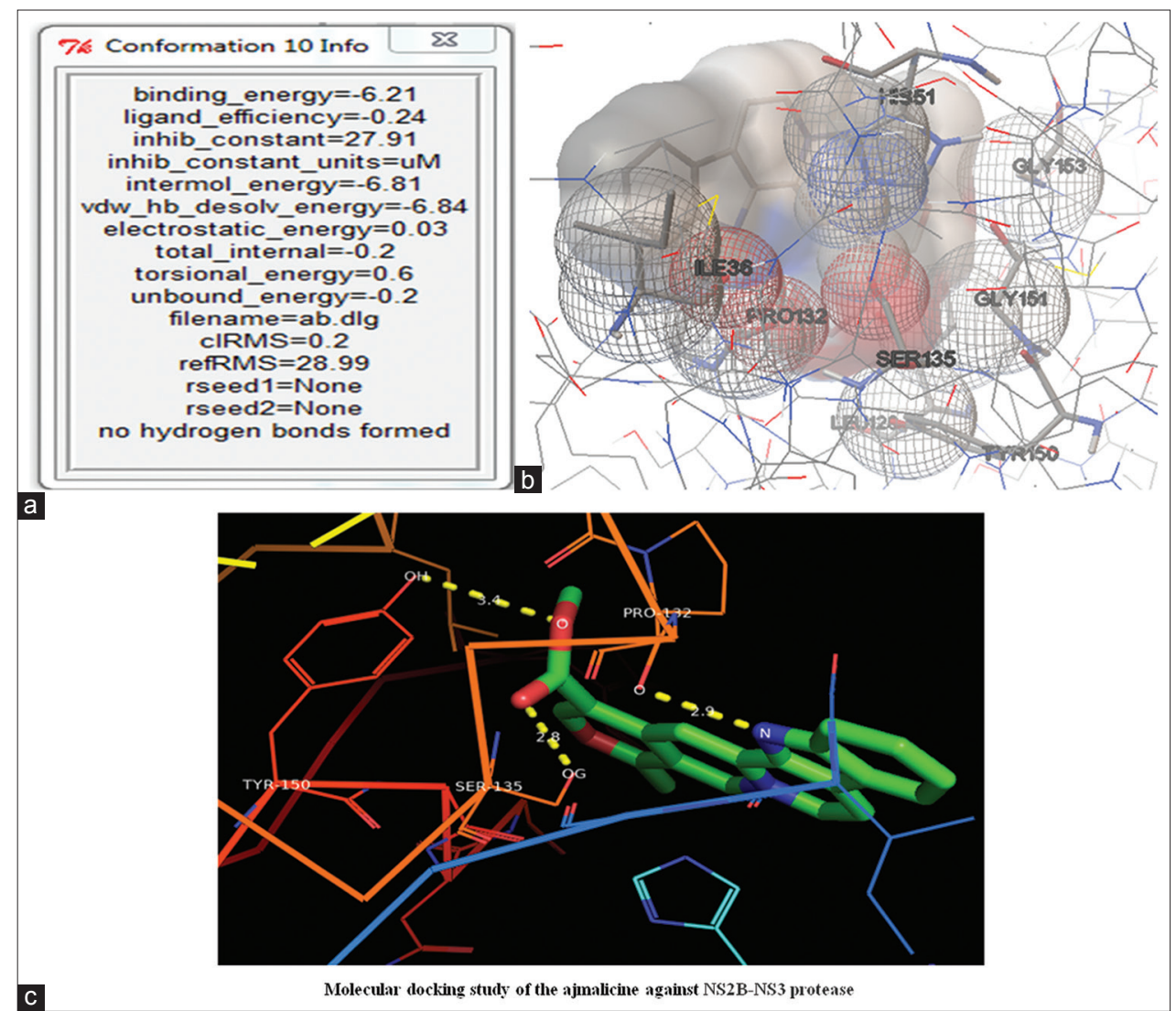

Fig. 4: Docking of NS2B-NS3 protease and ajmalicine (a) binding energy (b) interaction between NS2B-NS3 protease and ajmalicine as visualized using AutoDock (c) hydrogen bond forms between NS2B-NS3 protease and ajmalicine is visualized using PyMol 


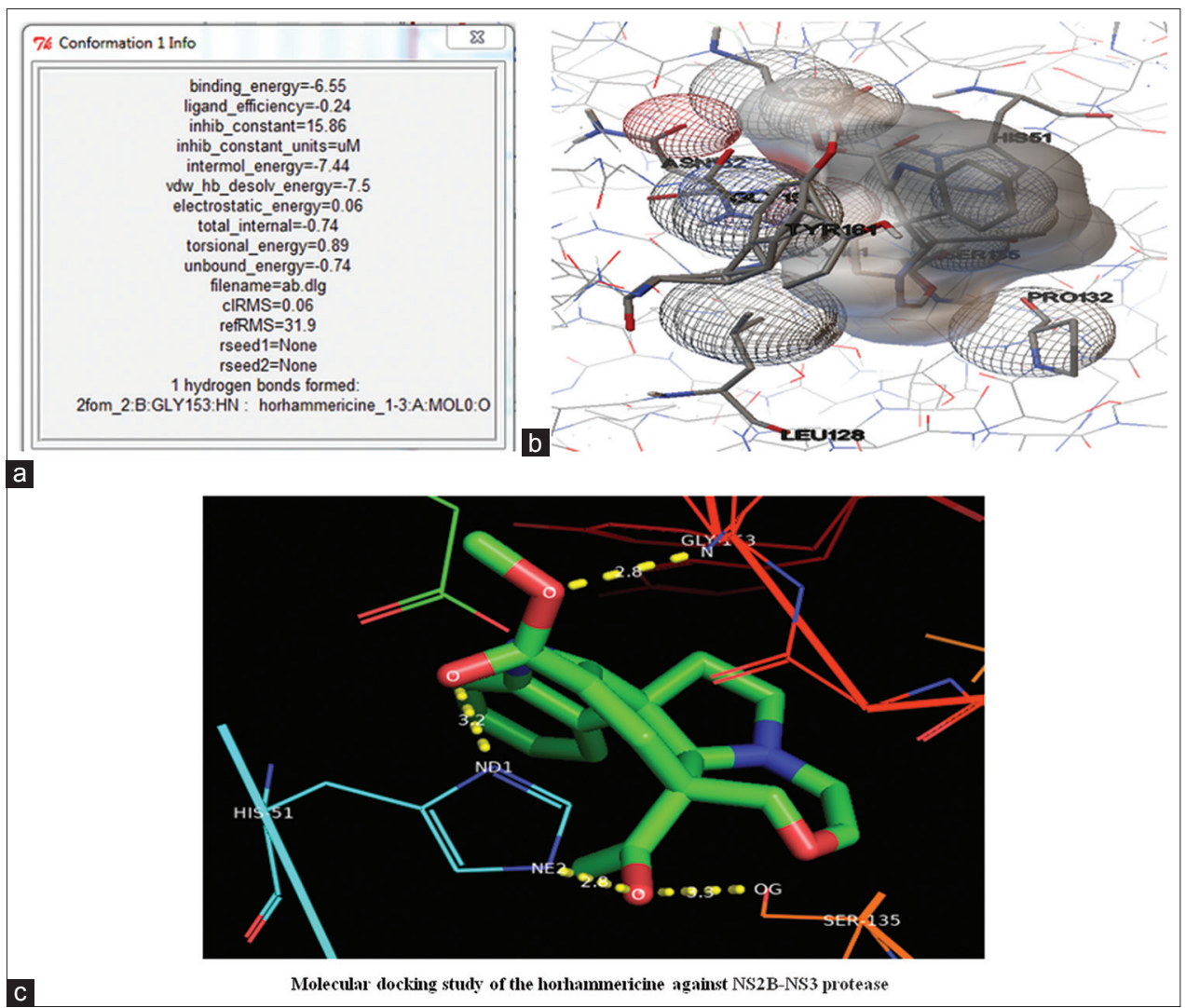

Fig. 5: Docking of NS2B-NS3 protease and horhammericine (a) binding energy (b) interaction between NS2B-NS3 protease and horhammericine as visualized using AutoDock (c) hydrogen bond forms between NS2B-NS3 protease and horhammericine is visualized using PyMol

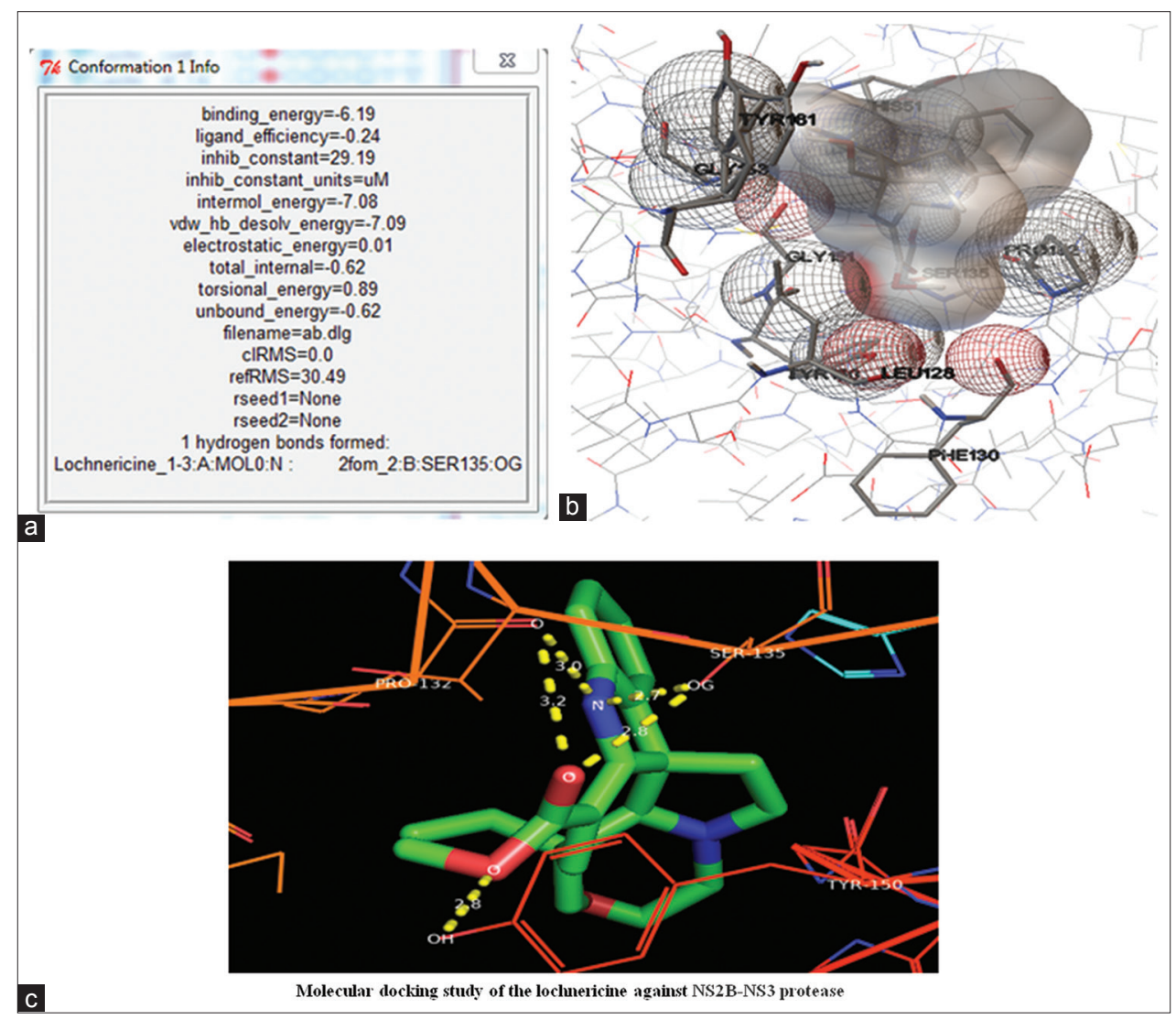

Fig. 6: Docking of NS2B-NS3 protease and lochnericine (a) binding energy (b) interaction between NS2B-NS3 protease and lochnericine as visualized using AutoDock (c) hydrogen bond forms between NS2B-NS3 protease and lochnericine is visualized using PyMol 


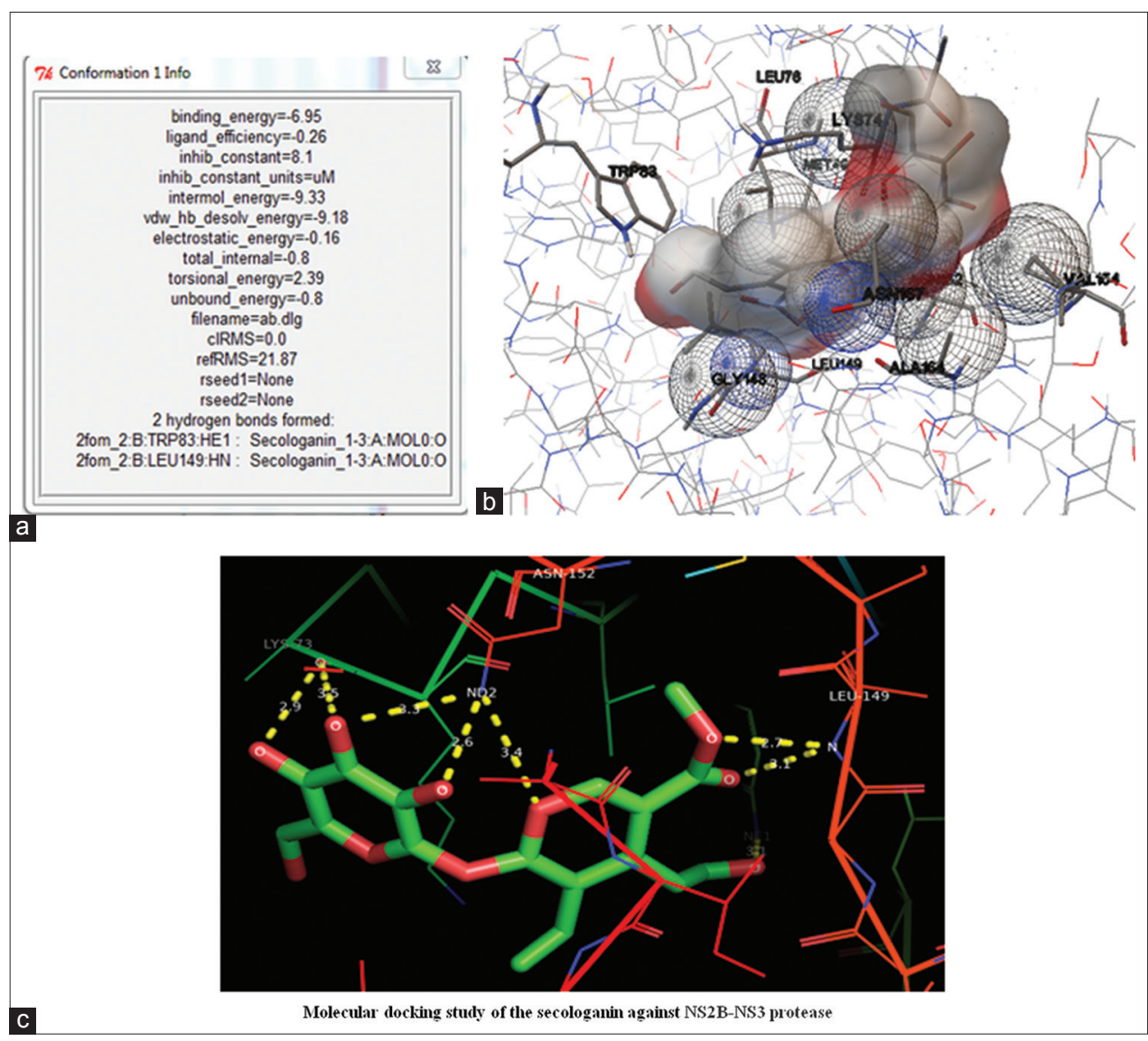

Fig. 7: Docking of NS2B-NS3 protease and secologanin (a) binding energy (b) interaction between NS2B-NS3 protease and secologanin as visualized using AutoDock (c) hydrogen bond forms between NS2B-NS3 protease and secologanin is visualized using PyMol

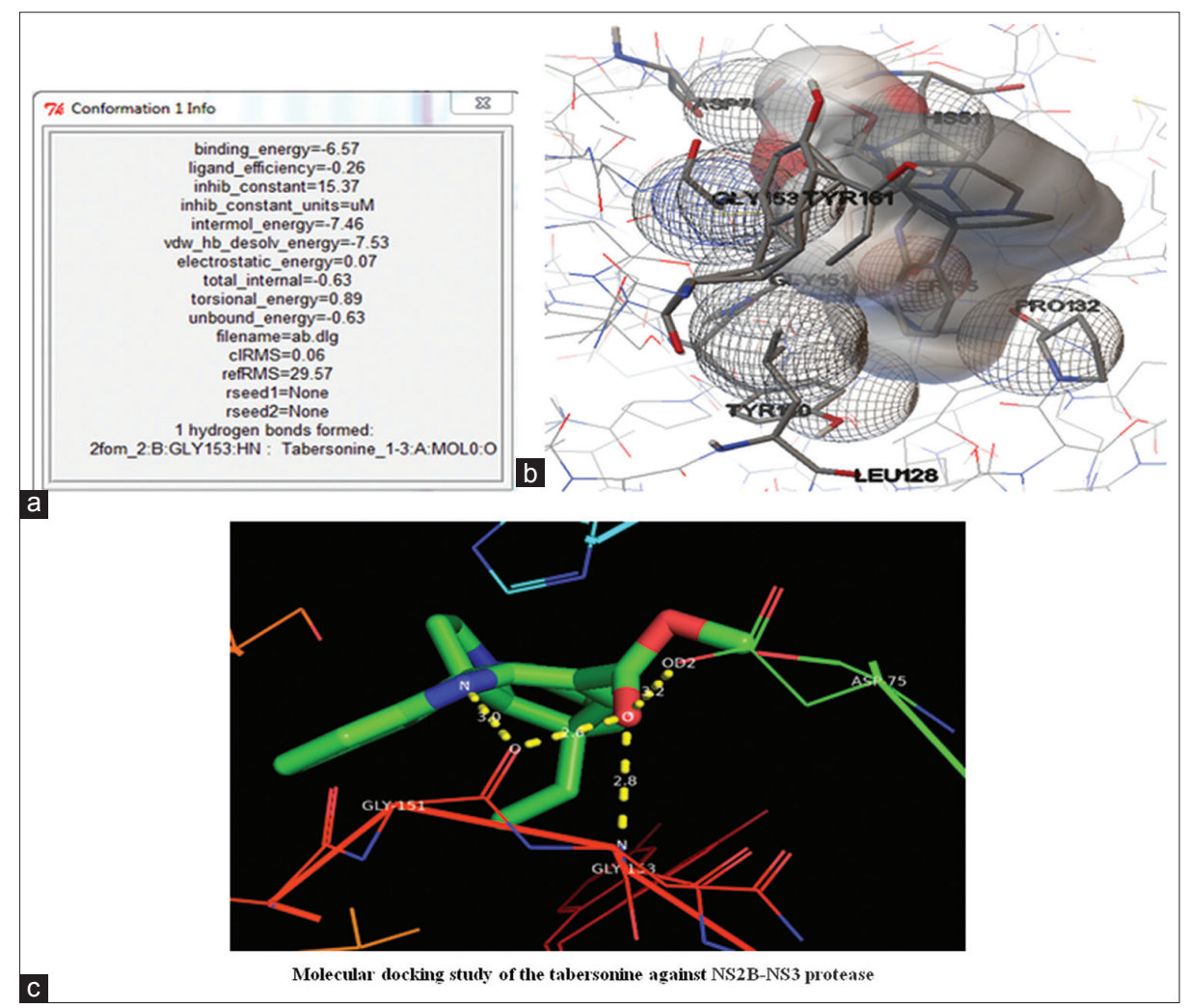

Fig. 8: Docking of NS2B-NS3 protease and tabersonine (a) binding energy (b) interaction between NS2B-NS3 protease and tabersonine as visualized using AutoDock (c) hydrogen bond forms between NS2B-NS3 protease and tabersonine is visualized using PyMol 


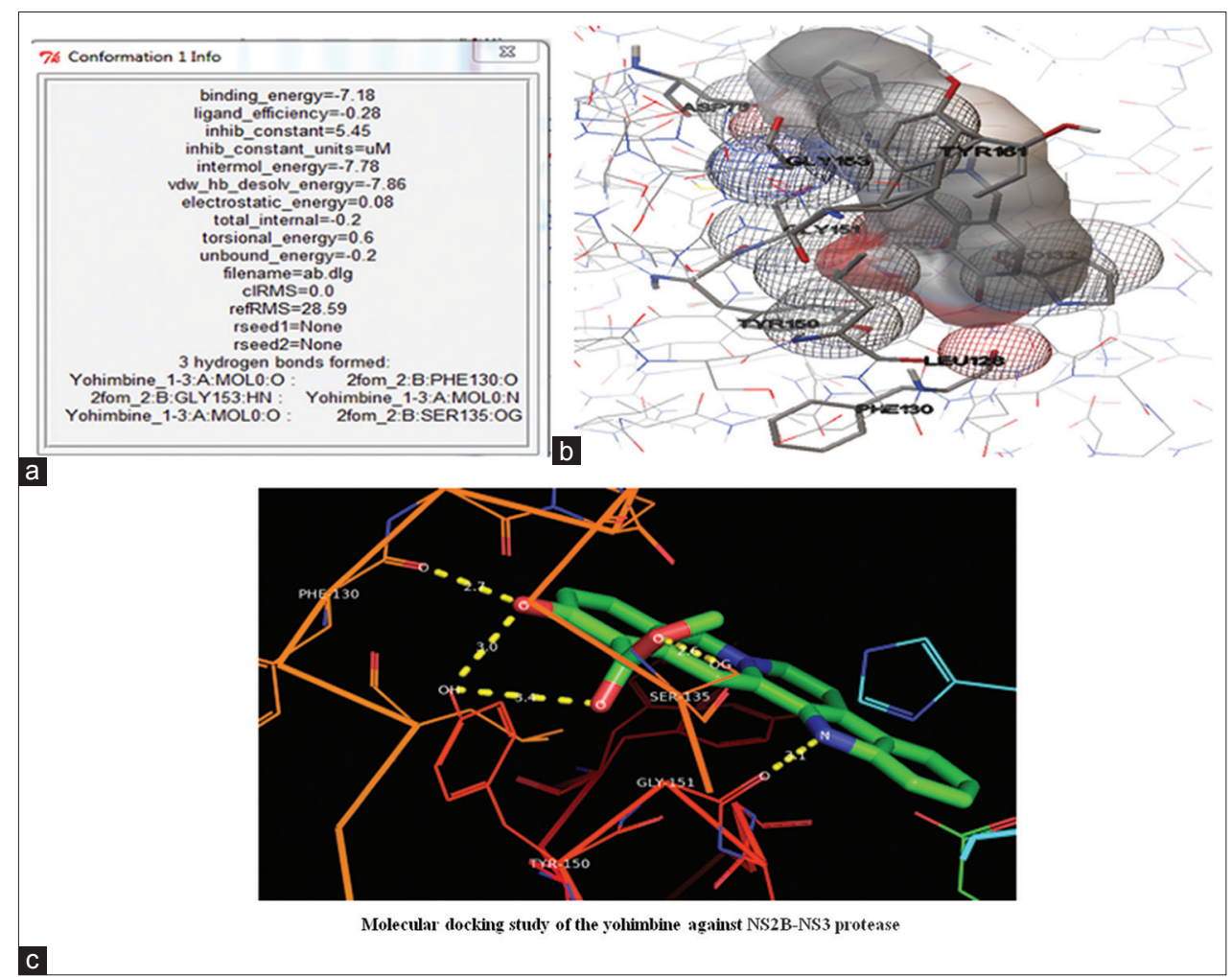

Fig. 9: Docking of NS2B-NS3 protease and yohimbine (a) binding energy (b) interaction between NS2B-NS3 protease and yohimbine as visualized using AutoDock (c) hydrogen bond forms between NS2B-NS3 protease and yohimbine is visualized using PyMol

receptor and ligand complex. The docked structures were analyzed and the interactions were seen. Hydrogen bond interactions and binding distance between donors and acceptors were measured for the best conformers. From the binding energy values, the anticancer activity of a ligand to the corresponding receptor was predicted [18].

\section{CONCLUSION}

Yohimbine compound are eco-friendly, safer, and cheaper for the treatment of dengue fever. The intention of this study is aimed to analyze the molecular docking studies on the target protein NS2B-NS3 protease which is responsible for dengue fever with the compound of vindoline, vindolinine, vindolidin, tabersonine, secologanin, yohimbine, Horhammericine, lochnericine, and ajmalicine which is evolved from C. roseus. Yohimbine is rich in alkaloid compound of medicinal value. The in silico docking studies were done by "AutoDock 4.2" software tool. Yohimbine is having the best binding score $(-7.18 \mathrm{Kcal} / \mathrm{mol})$ than the other eight compounds. Hence, it has been concluded yohimbine as a novel inhibitor for NS2B-NS3 protease in dengue fever.

\section{ACKNOWLEDGMENTS}

Authors are thankful to Bioinformatics Infrastructure Facility Centre (BIFC), Queen Mary's College, Chennai, and also thank Dr. R. Girija Coordinator, BIFC for their guidance and support.

\section{AUTHORS' CONTRIBUTIONS}

Dr. Girija. R Assistant Professor, Designed and Conducted the work focusing on medicinal aspects of C. roseus. Dr. Aruna. S Assistant Professor, analysis and framing work of the manuscript. Sangeetha. R Research Assistant developed the theory and performed the Docking Studies. All authors are considering the results and conclusion to the final manuscript.

\section{CONFLICTS OF INTEREST}

The authors are proclaim that they have no conflicts of interest.

\section{REFERENCES}

1. Gajalakshmi S, Vijayalakshmi S, Rajeswari VD. Pharmacological activities of Catharanthus roseus: A perspective review. Int J Pharm Bio Sci 2013;4:431-9.

2. Tiong SH, Looi CY, Hazni H, Arya A, Paydar M, Wong WF, et al. Antidiabetic and antioxidant properties of alkaloids from Catharanthus roseus (L.) G. Don. Molecules 2013;18:9770-84.

3. Yadav AK, Ambasta SK, Prasad SK, Trived MP. In vitro evaluation of anti bacterial property of Catharanthus roseus (Linn.) G. Don. Var. "Rosea" and "Alba". Int J Pharm Pharm Sci 2018;10:55-8.

4. Sain M, Sharma V. Catharanthus roseus (An anti-cancerous drug yielding plant) a review of potential therapeutic properties Int J Pure App Biosci 2013;1:139-42.

5. Sathiya S, Karthikeyan B, Jaleel CA, Azooz MM, Iqbal M. Antibiogram of Catharanthus roseus extracts. Glob J Mol Sci 2008;3:1-7.

6. Wang Q, Xing S, Pan Q, Yuan F, Zhao J, Tian Y, et al. Development of efficient Catharanthus roseus regeneration and transformation system using Agrobacterium tumefaciens and hypocotyls as explants BMC Biotechnol 2012;12:34.

7. Katiyar N, Nath R. In-silico protein-ligand docking studies against the NS5 methyltransferase protein of dengue virus. J Proteomics Bioinform 2018;11:127-34.

8. Sonagunalan S, Kayalvizhi S, Nageswari In silico Docking Study on Natural Compounds as Novel Inhibitors of Structural Viral Envelope Protein of Dengue Virus Type 4. Int J Sci Eng Technol 2016;4:481-91.

9. Geiss BJ, Stahla H, Hannah AM, Gari HH, Keenan SM. Focus on Flaviviruses: Current and future drug targets. Future Med Chem 2009;1:327.

10. Cragg GM, Newman DJ. Natural products: A continuing source of novel drug leads. Biochim Biophys Acta 2013;1830:3670-95.

11. Stevens AJ, Gahan ME, Mahalingam S, Keller PA. The medicinal chemistry of dengue fever. J Med Chem 2009;52:7911-26.

12. Tomlinson SM, Malmstrom RD, Watowich SJ. New approaches to structure-based discovery of dengue protease inhibitors. Infect Disord Drug Targ 2009;9:327-43.

13. Schüller A, Yin Z, Chia CS, Doan DN, Kim HK, Shang L, et al. Tripeptide inhibitors of dengue and West Nile virus NS2B-NS3 protease. Antiviral Res 2011;92:96-101.

14. Phong WY, Moreland NJ, Lim SP, Wen D, Paradkar PN, Vasudevan 
SG. Dengue protease activity: The structural integrity and interaction of NS2B with NS3 protease and its potential as a drug target. Biosci Rep 2011;31:399-409.

15. Luo D, Xu T, Watson RP, Scherer-Becker D, Sampath A, Jahnke W, et al. Insights into RNA unwinding and ATP hydrolysis by the Flavivirus NS3 protein. EMBO J 2008;27:3209-19.

16. Raja S, Kathiresan P, Sahu KS. In silico docking analysis of mangrove derived compounds against breast cancer protein (BRCA1). Int Multidiscip Res J 2011;1/1:9-12.

17. Pal S, Helix BD. An In silico drug designing approach to target the BRCA1 protein involved in breast cancer. 2016;6-1:761-765. Shilpi pal Helix 2016;6:761-765.

18. Premila J, Sangeetha R, Vijayalakshmi K. In silico docking analysis of capsaicin alkaloids from red chilli against breast cancer. Int J Curr Res
Aca Rev 2017:4:145-57.

19. Nithya G, Ilakkia A, Sakthisekaran D. In silico docking studies on the anti-cancer effect of thymoquinone on interaction with phosphatase and tensin homolog located on chromosome 10q23: A regulator of $\mathrm{PI} 3 \mathrm{k} /$ AKT pathway. Asian J Pharm Clin Res 2015;8:192-5.

20. Goodsell DS, Morris GM, Olson AJ. Automated docking of flexible ligands: Applications of auto dock. J Mol Recogn 1996;9:1-5.

21. Ali1 MH, Anwar S, Roy PK, Ashrafuzzaman M. Virtual screening for identification of small lead compound inhibitors of Nipah virus attachment glycoprotein. J Pharmacogenomics Pharmacoproteomics 2018;9:180.

22. Manigandan V, Ramanathan T. In silico docking of mangrove derived ligands against alzheimer's receptor proteins. Curr Res Neurosci 2014;4:18-24 